Historic, Archive Document

Do not assume content reflects current scientific knowledge, policies, or practices. 

Al $\cap-$

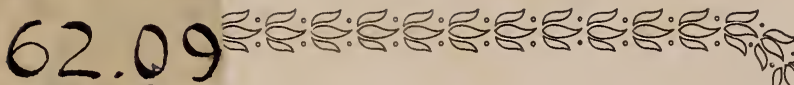

1902

iob

ido

i)

U'? the Wliolesale Th

Mngt?

9.0.

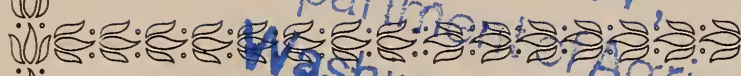

¿०0

200

100

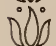

iोo

i.j

- FOR -

r9o Delivery.

SEEL

ington,

100

$\mathrm{O}^{\circ} \mathrm{O}$

i००

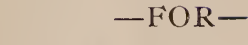

LIS"P

0.00

000

900

ن)

H. C. \& J. B. AGNEW

ilo

ilo

Agnew, California.

o. 0

000

0.00

000

000

900

000

ilo

900

000

000



Dido

D. Si Seed Ranches located at Agnew, 40 miles 000 ? Did south of San Francisco, in the famous Santa 000 Q Clara Valley, California and at Bethel, on 0 Isla Del Diablo, Contra Costa Co., California. 

H. C. \& J. B. Agnew,

\section{Seed Growers,}

Agnew, California.

Variety.

Price.

\section{ONION.}

V. Large Red Wethersfield............... .50 Vixtra Early Red Flat.................. .50 Southport Red Globe................... .55 Sillow Globe Danvers................ .50 Kound or Flat Danvers................ 45 Southport Yellow Globe .............. 50 Wrellow Strasburg (or Dutch) ........ .50 Urizetaker .............................. 65 Australian Brown .................... .50 Philadelphia Silverskin ............... 90 
2 H. L. \& J. B. AgNew.

Variety.

Price.

White Portugal ......................90

Southport IVhite Globe ................ Eixtra Early IVhite Pearl...............

\section{LETTTUCE.}

American Gathering..................20

All Year Round..................... "

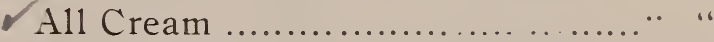

Big Bcston ........................... *

Black Seeded Simpson.............. "

Black Seeded 'Tennis Ball ............ "

Black Seeded Butter................. “

Boston Market .......................

California Cream Butter.............. "

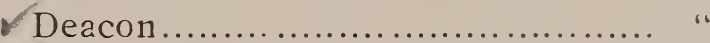

Early Curled Simpson (White seeded)

Early Stone...................... "

Eiarly White Cabbage................ "

Grand Rapids..................... "

VGolden Queen ....................... "

Hubbards Market................... " 


\section{Santa Clara, California.}

Variety.

Price.

Hanson

New Orleans.

$\checkmark$ New York (Henderson's Improved) 30

$\checkmark$ Philadelphia Butter.

.20

Prizehead .............................. "

Silesia Early Curled ..................."

$\checkmark$ St. Louis Butter...................... "

Salamander ............................ "

White Seeded Tennis Ball............ "

White Summer Cabbage............. “

\section{CELERY.}

Trhite Plume

Giant Pascal

Large Ribbed Kalamazoo.

\section{RADISH.}

Early Scarlet Turnip Rooted ......... . I 5

VEarly scarlet Turnip-Rooted-White

Tipped

Early Long Scarlet (Short top) 
+ H. L. \& J. B. AGNEN.

Variety.

Price.

Fiarly French Breakfast............. "

Improved Chartier................ "

\section{CARROT.}

$\checkmark$ Ox Heart or Guerande................. is Improved Long Orange............... " $\checkmark$ Half Long Orange or Danvers........ "

\section{SWEET PEA.}

America I $21 / 2$

American Seedlings. Apple Blossom.

Aurora

$\checkmark$ Black Knight.

$\checkmark$ Blanche Burpee.

$\checkmark$ Blanche Ferry, Extra Early.

Blushing Beauty.

$\checkmark$ Burpee's New Countess.

Captain of the Blues.

Captivation

Celestial

Chancellor 
Variety.

Price.

$\sqrt{ }$ Colonist

Coquette

Countess of Powis.

Countess of Cadogan.

25

Countess of Radnor ................. I $21 / 2$

Crown Jewel........................ "

Cupid Alice Eckford.................25

" Beauty....................... "

" Primrose....................... "

" Pink ......................... "

" White.......................... "

" Mixed.......................... "

Duchess of Sutherland............... I $21 / 2$

Duke of Sutherland.................. “

Duke of Westminster ................25

Dorothy Tennant .................. I $21 / 2$

Earliest of All.........................20

Emily Eckford....................... 1 $21 / 2$

Emily Henderson..................... “

Golden Gate ......................... " "

Gorgeous ............................. " 
Variety.

Price.

Gray Friar...................... I $21 / 2$

Grandiflora, Mixed.................. ro

Her Majesty ....................... I $21 / 2$

Hon. F. Bouverie...................25

Juanita ......................... I $21 / 2$

Katherine Tracy.................... "

Lady Mary Currie................... ،

Lady Nina Balfour................... ،

Lady Penzance...................... ،

Lady Skelmersdale................. .25

Lady Grisel Hamilton................ “

Lottie Hutchins.................... I $21 / 2$

Lottie Eckford ..................... "

Lovely .............................. ،

Maid of Honor ...................... "

Mars .............................. "

Meteor.............................. "

Modesty .......................... "

Monarch............................ "

Mrs. Dugdale.......................25

Mrs. Eckford $\ldots \ldots \ldots \ldots \ldots \ldots \ldots \ldots$ I $21 / 2$ 


\section{Santa Clara, California.}

Variety.

Price.

Mrs. Joseph Chamberlain........... I 2 1/2

Navy Blue ........................25

Oriental ......................... I $2 \dot{1} / 2$

Othello .............................25

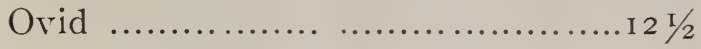

Pink Friar .......................... ،

Prima Donna....................... ،

Prince Edward of York.............. "

Prince of Wales ...................... "

Queen Victoria ..................... “"

Ramona ............................. “

Red Riding Hood.................... ،

Royal Rose.......................... ،

Sadie Burpee......................25

Salopian ........................ I $21 / 2$

Sensation ........................... "

Shahzada ............................. "

Stanley ............................ ،

Stella Morse.............. .......... ،

Triumph........................... ،

Venus........................... . " 


\section{American Sugar Beet Seed.}

We are not placing any of our American Sugar Beet Seed stock on the market, as we have it all contracted in advance and we grow only for Factory orders.

Note-While we exercise the greatest care to have all seeds pure and reliable, we do not give any warranty, expressed or implied.

All contracts are subject to crop, as we are strictly growers and not merchants. F. O. B. California.

$$
\text { H. C. \& J. B. AGNEW. }
$$




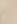




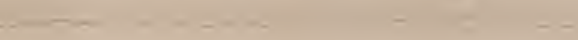

